\title{
A novel approach to solve nonlinear Fredholm integral equations of the second kind
}

\author{
Hu Li and Jin Huang
}

${ }^{*}$ Correspondence: lihuxiwangzhixing@163.com School of Mathematical sciences, University of Electronic Science and Technology of China, Chengdu 611731, Sichuan, People's Republic of China

\begin{abstract}
In this paper, we present a novel approach to solve nonlinear Fredholm integral equations of the second kind. This algorithm is constructed by the integral mean value theorem and Newton iteration. Convergence and error analysis of the numerical solutions are given. Moreover, Numerical examples show the algorithm is very effective and simple.
\end{abstract}

Keywords: A novel approach, Nonlinear Fredholm integral equations, Integral mean value theorem, Newton iteration

\section{Background}

Integral equations have several applications in Physics and Engineering. However, these occur nonlinearly. In particular, nonlinear integral equations arise in fluid mechanics, biological models, solid state physics, kinetics in chemistry etc. In most cases, it is difficult to solve them, especially analytically.

In the past several years, the nonlinear integral equations have been solved numerically by several workers, utilizing various approximate methods (see Atkinson and Potra 1988; Atkinson and Flores 1993; Babolian and Shahsavaran 2009; Lepik and Tamme 2007; Saberi-Nadjafi and Heidari 2010; Aziz and Islam 2013; Maleknejad and Nedaiasl 2011).

In the present work, we have developed a novel approach to solve nonlinear Fredholm integral equations of the second. This algorithm is obtained by integral mean value theorem and Newton iteration. We consider the nonlinear Fredholm integral equations, given as follows:

$$
u(x)=f(x)+\int_{a}^{b} K(x, y) g(u(y)) d y, \quad x \in[a, b],
$$

where $f(x)$ is a known continuous function defined on $[a, b]$ and $g(u(y))$ is a nonlinear function defined on $[a, b]$. The nonlinear integral operator $k$ is defined as follows:

$$
(k g(u))(x)=\int_{a}^{b} K(x, y) g(u(y)) d y, \quad x \in[a, b],
$$

\section{Springer}

(c) $2016 \mathrm{Li}$ and Huang. This article is distributed under the terms of the Creative Commons Attribution 4.0 International License (http://creativecommons.org/licenses/by/4.0/), which permits unrestricted use, distribution, and reproduction in any medium, provided you give appropriate credit to the original author(s) and the source, provide a link to the Creative Commons license, and indicate if changes were made. 
and $k$ is compact on $C[a, b]$ into $C[a, b]$ with continuous kernel $K(x, y)$. Then (1) is equivalent to the operator form as follows:

$$
u-k g(u)=f .
$$

This paper is organized as follows: In section "A novel numerical method", based on the idea of the integral mean value theorem, a novel numerical method is given. In section "Convergence and error analysis", we address the convergence and error analysis of the numerical solutions. In section "Description of Newton iteration and a novel algorithm", Newton iteration is introduced and a novel algorithm is given. In section "Numerical results", numerical examples are carried out.

\section{A novel numerical method}

In order to obtain a novel numerical method, we firstly introduce the integral mean value theorem, is given as follows:

Theorem 1 If $s(x)$ is continuous on the closed interval $[a, b]$, there is a number $c \in[a, b]$ so that

$$
M(s)=\int_{a}^{b} s(x) d x=(b-a) s(c) .
$$

Let $h=(b-a) / n, n \in N$ be the mesh with $x_{k}=a+k h, k=0, \ldots, n$. By (4), we can construct a sequence of quadrature formula as

$$
M\left(s, c_{k}\right)=\sum_{k=0}^{n-1} \int_{x_{k}}^{x_{k+1}} s(x) d x=h \sum_{k=0}^{n-1} s\left(x_{k}+h c_{k}\right), \quad 0<c_{k}<1,
$$

where $c_{k},(k=0, \ldots, n-1)$ are constants.

We apply (5) to the integral operator $K$ and get

$$
(k g(u))(x)=h \sum_{k=0}^{n-1} K\left(x, x_{k}+h c_{k}(x)\right) g\left(u\left(x_{k}+h c_{k}(x)\right)\right), \quad x \in[a, b],
$$

where the unknown function $c_{k}(x),(k=0, \ldots, n-1)$ are dependent on the variable $x$ and $0<c_{k}(x)<1$. Especially, Let $c_{k}(x)=c_{k}$ be constants. We can obtain Nyström approximation with a high accuracy, is given as follows:

$$
\left(k_{n} g(u)\right)(x)=h \sum_{k=0}^{n-1} K\left(x, x_{k}+h c_{k}\right) g\left(u\left(x_{k}+h c_{k}\right)\right), \quad x \in[a, b], \quad 0<c_{k}<1 .
$$

Thus we obtain the numerical approximate form of (3)

$$
u_{n}-k_{n} g\left(u_{n}\right)=f \text {. }
$$

Obviously, Eq. (8) is a nonlinear equations system. Once $u_{n}$ is get, we obtain $u(x), x \in[a, b]$ by $(3)$. 


\section{Convergence and error analysis}

We give the convergence analysis of (8) and have a theorem as follows:

Theorem 2 If the function $K(x, y)$ is continuous on $[a, b] \times[a, b]$ and $g(x)$ is continuous on $[a, b]$, they satisfy the following Lipschitz conditions

$$
\begin{aligned}
& \left\|K\left(x, y_{1}\right)-K\left(x, y_{2}\right)\right\|_{\infty} \leq L_{1}\left\|y_{1}-y_{2}\right\|_{\infty}, \\
& \left\|g\left(x_{1}\right)-g\left(x_{2}\right)\right\|_{\infty} \leq L_{2}\left\|x_{1}-x_{2}\right\|_{\infty}, \\
& \left\|u\left(x_{1}\right)-u\left(x_{2}\right)\right\|_{\infty} \leq L_{3}\left\|x_{1}-x_{2}\right\|_{\infty},
\end{aligned}
$$

with the constants $L_{1,2,3}>0$, the sequence $\left(k_{n} g(u)\right)(x)$ of quadrature formula is convergent. That is, we have

$$
\left(k_{n} g(u)\right)(x) \rightarrow(k g(u))(x)=\int_{a}^{b} K(x, y) g(u(y)) d y, \quad n \rightarrow \infty .
$$

Proof By (6) and (7), we easily get

$$
\begin{aligned}
&\left\|\left(k_{n} g(u)\right)(x)-(k g(u))(x)\right\|_{\infty} \\
& \leq h \sum_{k=0}^{n-1}\left\|K\left(x, x_{k}+h c_{k}\right) g\left(u\left(x_{k}+h c_{k}\right)\right)-K\left(x, x_{k}+h c_{k}(x)\right) g\left(u\left(x_{k}+h c_{k}(x)\right)\right)\right\|_{\infty} \\
&= h \sum_{k=0}^{n-1} \| K\left(x, x_{k}+h c_{k}\right) g\left(u\left(x_{k}+h c_{k}\right)\right)-K\left(x, x_{k}+h c_{k}\right) g\left(u\left(x_{k}+h c_{k}(x)\right)\right) \\
&+K\left(x, x_{k}+h c_{k}\right) g\left(u\left(x_{k}+h c_{k}(x)\right)\right)-K\left(x, x_{k}+h c_{k}(x)\right) g\left(u\left(x_{k}+h c_{k}(x)\right)\right) \|_{\infty} \\
& \leq h \sum_{k=0}^{n-1}\left\|K\left(x, x_{k}+h c_{k}\right) g\left(u\left(x_{k}+h c_{k}\right)\right)-K\left(x, x_{k}+h c_{k}\right) g\left(u\left(x_{k}+h c_{k}(x)\right)\right)\right\|_{\infty} \\
&+h \sum_{k=0}^{n-1}\left\|K\left(x, x_{k}+h c_{k}\right) g\left(u\left(x_{k}+h c_{k}(x)\right)\right)-K\left(x, x_{k}+h c_{k}(x)\right) g\left(u\left(x_{k}+h c_{k}(x)\right)\right)\right\|_{\infty} \\
& \leq h^{2}\left[L_{2} L_{3} \max _{a \leq x, y \leq b}|K(x, y)|+L_{1}\|g(u(x))\|_{\infty}\right] \sum_{k=0}^{n-1}\left\|c_{k}-c_{k}(x)\right\|_{\infty} \\
& \leq \frac{(b-a)^{2}}{n}\left[L_{2} L_{3} \max _{a \leq x, y \leq b}|K(x, y)|+L_{1}\|g(u(x))\|_{\infty}\right],
\end{aligned}
$$

where $0<c_{k}<1$ and $0<c_{k}(x)<1$. We have $\left\|\left(k_{n} g(u)\right)(x)-(k g(u))(x)\right\|_{\infty} \rightarrow 0, n \rightarrow \infty$, and the proof of the theorem is completed.

From Theorem 2, we can get a corollary as follows:

Corollary 1 Under the assumption of Theorem 2, the error of the approximate solutions in (8) can be estimated, is given as follows:

$$
\left\|u_{n}(x)-u(x)\right\|_{\infty} \leq \frac{(b-a)^{2}}{n}\left[L_{2} L_{3} \max _{a \leq x, y \leq b}|K(x, y)|+L_{1}\|g(u(x))\|_{\infty}\right] .
$$




\section{Description of Newton iteration and a novel algorithm}

We shall give Newton iteration to solve nonlinear equations. For convenience, we denote

$$
\Psi(z)=\left(\varphi_{0}(z), \ldots, \varphi_{n-1}(z)\right),
$$

where $z=\left(z_{0}, \ldots, z_{n-1}\right)^{T}=u_{n}$, and

$$
\varphi_{i}(z)=z_{i}-h \sum_{j=0}^{n-1} K_{i, j} g\left(z_{j}\right)-f_{i}, \quad i=0, \ldots, n-1
$$

with $K_{i, j}=K\left(x_{i}+h c_{i}, x_{j}+h c_{j}\right)$. Then, (8) can be rewritten as

$$
\Psi(z)=0 .
$$

The Jaccobi matrix of $\Psi(z)$ is

$$
A(z)=\Psi^{\prime}(z)=\left(\partial_{j} \varphi_{i}(z)\right)_{n \times n} .
$$

So New iteration is constructed

$$
z^{l+1}=\omega\left(z^{l}\right), \omega(z)=z-(A(z))^{-1} \Psi(z), \quad l=0,1,2, \ldots
$$

Lemma 1 [Ostrowski see Ortege and Kheinboldt (1970)] Suppose there is a fixed point $z^{*} \in \operatorname{int}(D)$ of the mapping: $\omega: D \subset R^{n} \rightarrow R^{n}$ and the F-derivation of $\omega$ at point $z^{*}$ exists. If the spectral radius of $\omega^{\prime}\left(z^{*}\right)$ satisfies

$$
\rho\left(\omega^{\prime}\left(z^{*}\right)\right)=\delta<1 .
$$

Then, there is an open ball $S=S\left(z^{*}, \delta_{0}\right) \subset D$ that for $z^{0} \in S$, the iterative sequence (14) is stable and convergent to $z^{*}$.

Lemma 2 [see Ortege and Kheinboldt (1970)] Suppose $A, C \in L\left(R^{n}\right),\left\|A^{-1}\right\|<\beta$, $\|A-C\|<\alpha, \alpha \beta<1$, then $C$ is invertible and $\left\|C^{-1}\right\|<\beta /(1-\alpha \beta)$.

Theorem 3 Suppose $\Psi: D \subset R^{n} \rightarrow R^{n}$ is F-derivative, and $z^{*}$ satisfies equation $\Psi(z)=0 . A: S \subset D \rightarrow L\left(R^{n}\right)$ is continuous and invertible at $z^{*}$, where $S$ is the neighborhood of $z^{*}$. Then, there is a close ball $\bar{S}=\bar{S}\left(z^{*}, \delta\right) \subset S$ that $\Omega$ is F-derivative at $z^{*}$ :

$$
\omega^{\prime}\left(z^{*}\right)=I-\left(A\left(z^{*}\right)\right)^{-1} \Psi^{\prime}\left(z^{*}\right) .
$$

Proof Let $\beta=\left\|\left(A\left(z^{*}\right)\right)^{-1}\right\|>0$. Since $A\left(z^{*}\right)$ is invertible, and $A(z)$ is continuous at $z^{*}$, for $0<\varepsilon<(2 \beta)^{-1}, \exists \delta>0$, when $z \in \bar{S}\left(z^{*}, \delta\right)$, there is $\left\|A(z)-A\left(z^{*}\right)\right\|<\varepsilon$. According to Lemma $2,(A(z))^{-1}$ exists and $\left\|(A(z))^{-1}\right\| \leq \beta /(1-\varepsilon \beta)$ for any $z \in \bar{S}$. So we construct the function

$$
\omega(z)=z-(A(z))^{-1} \Psi(z), \quad z \in \bar{S} .
$$

Since $\Psi(z)$ is derivative at $z^{*}, \exists \delta>0$. When $z \in \bar{S}\left(z^{*}, \delta\right)$, we obtain an inequality by the definition of the F-derivation: 


$$
\left\|\Psi(z)-\Psi\left(z^{*}\right)-\Psi^{\prime}\left(z^{*}\right)\left(z-z^{*}\right)\right\| \leq \varepsilon\left\|z-z^{*}\right\| .
$$

Consider the derivation of $\omega(z)$

$$
\begin{aligned}
& \left\|\omega(z)-\omega\left(z^{*}\right)-\left[I-\left(A\left(z^{*}\right)\right)^{-1} \Psi^{\prime}\left(z^{*}\right)\right]\left(z-z^{*}\right)\right\| \\
& =\left\|-(A(z))^{-1} \Psi(z)-\left(A\left(z^{*}\right)\right)^{-1} \Psi^{\prime}\left(z^{*}\right)\left(z-z^{*}\right)\right\| \\
& \leq\left\|(A(z))^{-1}\left(A\left(z^{*}\right)-A(z)\right)\left(A\left(z^{*}\right)\right)^{-1} \Psi^{\prime}\left(z^{*}\right)\left(z-z^{*}\right)\right\| \\
& \quad+\left\|(A(z))^{-1}\left(\Psi(z)-\Psi\left(z^{*}\right)-\Psi^{\prime}\left(z^{*}\right)\left(z-z^{*}\right)\right)\right\| \\
& \leq\left(2 \beta^{2} \varepsilon\left\|\Psi^{\prime}\left(z^{*}\right)\right\|+2 \beta \varepsilon\right) \leq c \varepsilon\left\|z-z^{*}\right\|,
\end{aligned}
$$

where $c=2 \beta\left(\beta\left\|\Psi^{\prime}\left(z^{*}\right)\right\|+1\right)$. According to the definition of the F-derivation, we obtain the the F-derivation of $\omega$ at $z^{*}$

$$
\omega^{\prime}\left(z^{*}\right)=I-\left(A\left(z^{*}\right)\right)^{-1} \Psi^{\prime}\left(z^{*}\right) .
$$

Using the definition of the matrix $A$ in (13), we have $\rho\left(\omega^{\prime}\left(z^{*}\right)\right)=0<1$. According to Lemma 1 , the iterative sequence is stable and convergent to $z^{*}$.

In what follows, in order to give the numerical solutions with more stability, we provide a novel algorithm (see Zhong 2013).

Step 1 Take $n$ and Let $x_{k}=a+h k,(k=0, \ldots, n-1)$ with $h=(b-a) / n$.

Step 2 Let $c_{k}=c,(k=0, \ldots, n-1)$ and randomly choose a series of $\sigma_{i}$ so that $0<c=\sigma_{i}<1,(i=0,1, \ldots, m)$.

Step 3 Solve the nonlinear system by Newton iteration

$$
u_{n}^{j}-h \sum_{k=0}^{n-1} K\left(x_{j}+h \sigma_{i}, x_{k}+h \sigma_{i}\right) g\left(u_{n}^{k}\right)=f\left(x_{j}+h \sigma_{i}\right) .
$$

Step 4 Get the approximate solutions

$$
u_{n}\left(x, \sigma_{i}\right)=f(x)+h \sum_{k=0}^{n-1} K\left(x, x_{k}+h \sigma_{i}\right) g\left(u_{n}^{k}\right) .
$$

Step 5 Let the mean value of $u_{n}\left(x, \sigma_{i}\right)$ be the last approximate solution

$$
u_{n}(x)=\sum_{i=0}^{m} \frac{u_{n}\left(x, \sigma_{i}\right)}{m+1}
$$

\section{Numerical results}

In this section, the theoretical results of the previous section are used for some numerical examples. 
Example 1 The following nonlinear integral equation is considered

$$
u(x)=x \int_{0}^{1} y \sqrt{u(y)} d y+2-\frac{1}{3}(2 \sqrt{2}-1) x-x^{2}
$$

with $0<x<1$ and the exact solution $u(x)=2-x^{2}$.

For the sake of simplicity, we choose $\sigma_{i}=i / 10,(i=0,1, \ldots, 10)$. Table 1 shows the three kinds results by using the methods in Lepik and Tamme (2007), Aziz and Islam (2013), and the present method, respectively. Figure 1 shows the comparison of approximate and exact solutions with $n=128$ and Fig. 2 presents the error curve on $[0,1]$ with $n=128$.

Example 2 The following nonlinear integral equation is considered

$$
u(x)=\frac{1}{5} \int_{0}^{1} \cos (\pi x) \sin (\pi y)[u(y)]^{3} d y+\sin (\pi x),
$$

with $0<x<1$ and the exact solution $u(x)=\sin (\pi x)+\frac{1}{3}(20-\sqrt{391}) \cos (\pi x)$.

Table 1 Absolute errors for Example 1

\begin{tabular}{llllll}
\hline $\boldsymbol{x}$ & $\mathbf{n = 8}$ & $\boldsymbol{n = 1 6}$ & $\boldsymbol{n = 3 2}$ & $\boldsymbol{n = 6 4}$ & $\boldsymbol{n = 1 2 8}$ \\
\hline 0.2 & $1.63 \mathrm{e}-3$ & $4.09 \mathrm{e}-4$ & $9.94 \mathrm{e}-5$ & $2.48 \mathrm{e}-6$ & $6.21 \mathrm{e}-7$ \\
0.4 & $3.27 \mathrm{e}-3$ & $8.18 \mathrm{e}-4$ & $1.99 \mathrm{e}-4$ & $4.97 \mathrm{e}-6$ & $1.24 \mathrm{e}-6$ \\
0.6 & $4.90 \mathrm{e}-3$ & $1.23 \mathrm{e}-3$ & $2.98 \mathrm{e}-4$ & $7.45 \mathrm{e}-6$ & $1.86 \mathrm{e}-6$ \\
0.8 & $6.54 \mathrm{e}-3$ & $1.64 \mathrm{e}-3$ & $3.97 \mathrm{e}-4$ & $9.94 \mathrm{e}-6$ & $2.48 \mathrm{e}-6$ \\
Results in Aziz and Islam (2013) & $1.0 \mathrm{e}-3$ & $2.6 \mathrm{e}-4$ & $6.6 \mathrm{e}-5$ & $1.7 \mathrm{e}-5$ & $4.2 \mathrm{e}-6$ \\
Results in Lepik and Tamme (2007) & $2.7 \mathrm{e}-3$ & $1.1 \mathrm{e}-3$ & $3.7 \mathrm{e}-4$ & $1.1 \mathrm{e}-4$ & $3.1 \mathrm{e}-5$ \\
\hline
\end{tabular}

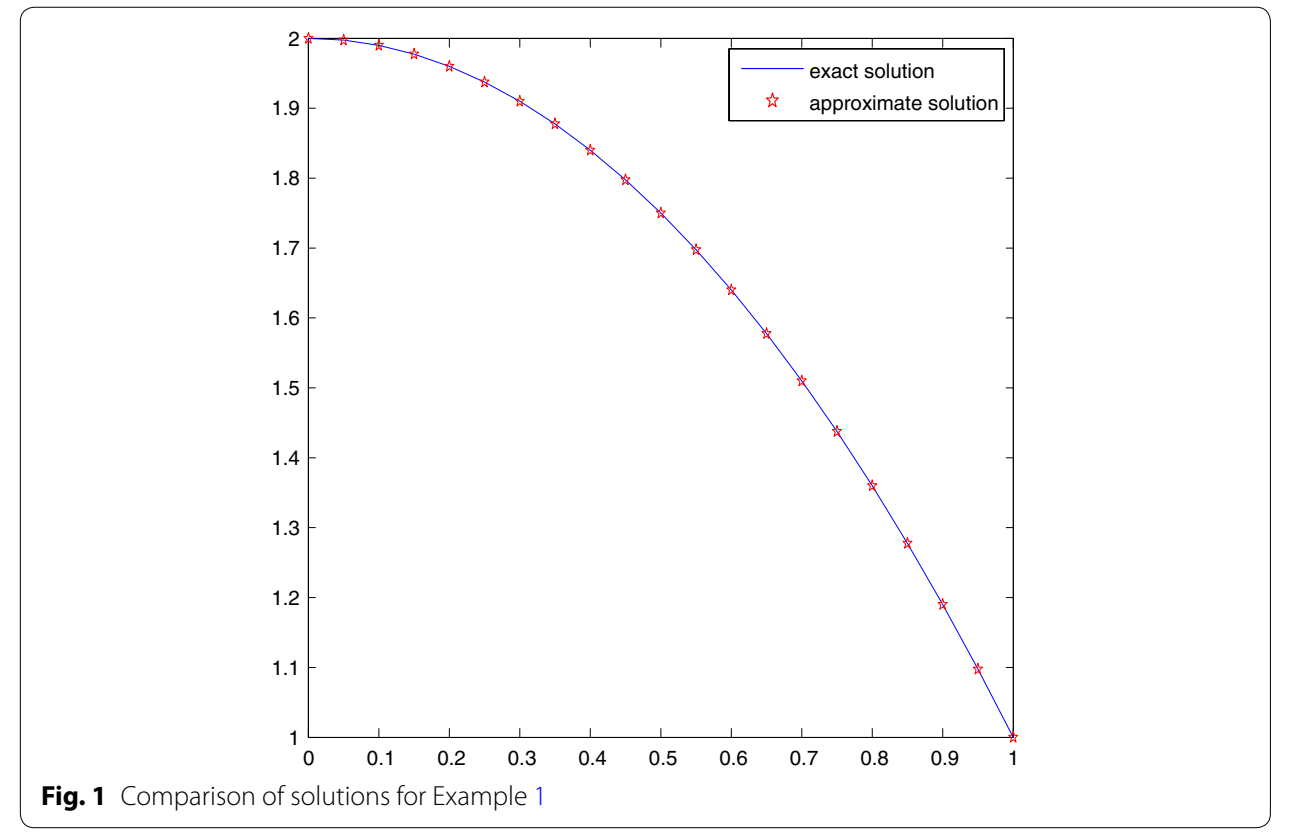




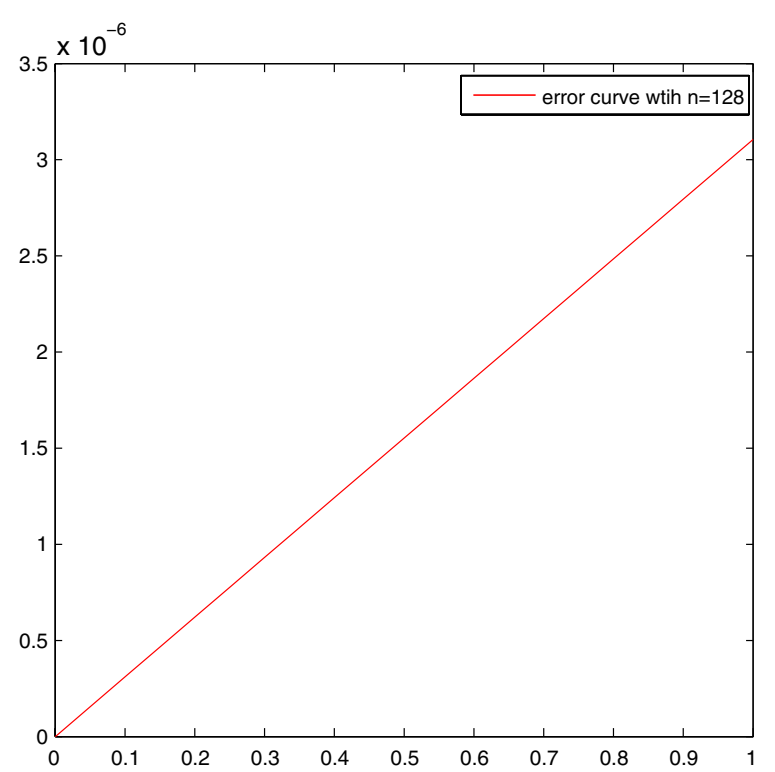

Fig. 2 The error curve for Example 1

Table 2 Absolute errors for Example 2

\begin{tabular}{lllll}
$\boldsymbol{x}$ & $\begin{array}{l}\text { Results in Saberi-Nadjafi } \\
\text { and Heidari (2010) }\end{array}$ & $\begin{array}{l}\text { s-results in Maleknejad } \\
\text { and Nedaiasl (2011) }\end{array}$ & $\begin{array}{l}\text { d-results in Maleknejad } \\
\text { and Nedaiasl (2011) }\end{array}$ & $\begin{array}{l}\text { Present } \\
\text { method }\end{array}$ \\
\hline 0 & $4.98 \mathrm{e}-2$ & $4.15 \mathrm{e}-7$ & $1.40 \mathrm{e}-10$ & $3.19 \mathrm{e}-16$ \\
0.3 & $2.92 \mathrm{e}-2$ & $6.22 \mathrm{e}-6$ & $1.25 \mathrm{e}-8$ & $3.33 \mathrm{e}-16$ \\
0.6 & $1.54 \mathrm{e}-2$ & $5.85 \mathrm{e}-6$ & $9.54 \mathrm{e}-9$ & $1.11 \mathrm{e}-16$ \\
0.9 & $4.73 \mathrm{e}-2$ & $1.66 \mathrm{e}-5$ & $1.61 \mathrm{e}-8$ & $3.89 \mathrm{e}-16$ \\
\hline
\end{tabular}

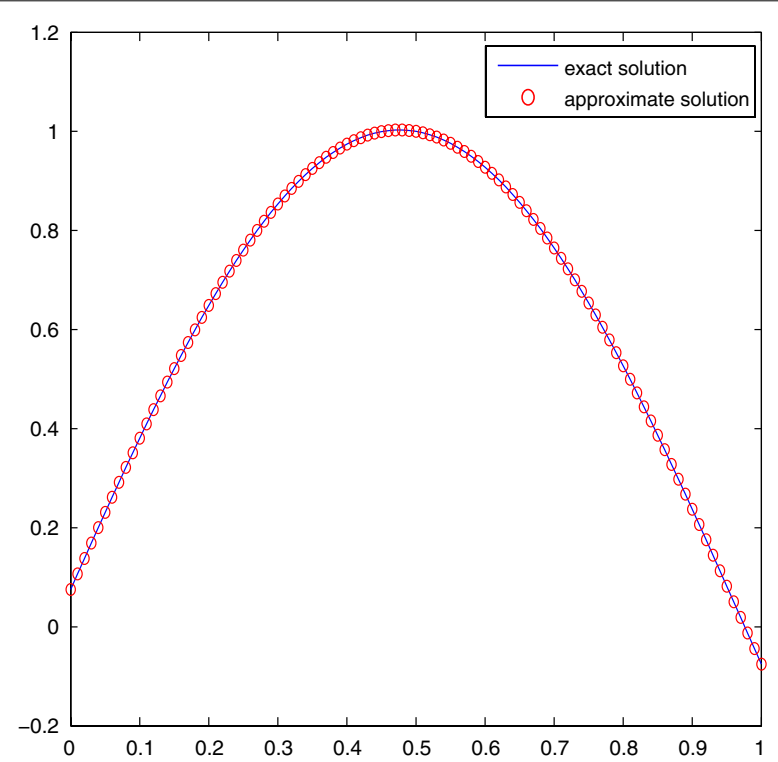

Fig. 3 Comparison of solutions for Example 2 
We take $n=25$ along with $\mathrm{h}=1 / 25$ and get $x_{k}=k / 25,(k=0,1, \ldots, 24)$. For the sake of simplicity, $\sigma_{i}$ is given as $i / 10,(i=0,1, \ldots, 10)$. Table 2 shows the four kinds results by using Newton-Kantorovich-quadrature method in Saberi-Nadjafi and Heidari (2010), the SE-Sinc method in Maleknejad and Nedaiasl (2011), the DE-Sinc method in Maleknejad and Nedaiasl (2011), and the present method, respectively. Figure 3 shows the comparison of approximate and exact solutions with $n=25$ and Fig. 4 presents the error curve on $[0,1]$ with $n=25$.

Example 3 The following nonlinear integral equation is considered

$$
u(x)+\int_{0}^{1} x e^{u(y)} d y=x e^{1},
$$

with $0<x<1$ and the exact solution $u(x)=x$.

In Table 3, we choose $\sigma_{i}=i / 10,(i=0,1, \ldots, 10)$. In Table 4 , we choose $\sigma_{i}=0,1 / 2,1$, and $r_{i},(i=0,1, \ldots, 10)$, respectively. $r_{i},(i=0,1, \ldots, 10)$ are randomly selected. Table 3 shows the numerical results by using the present method, and Table 4 shows the choice of $\sigma_{i}$ has a great influence on the accuracy of numerical solutions.

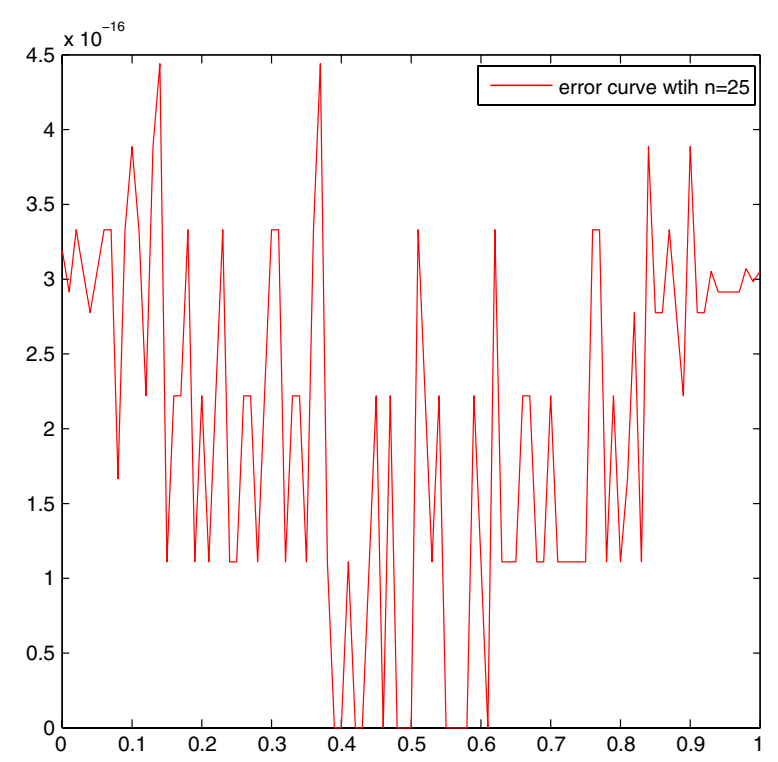

Fig. 4 The error curve for Example 2

Table 3 Absolute errors for Example 3

\begin{tabular}{llllll}
\hline $\boldsymbol{x}$ & $\boldsymbol{n}=\mathbf{4}$ & $\boldsymbol{n}=\mathbf{8}$ & $\boldsymbol{n}=\mathbf{1 6}$ & $\boldsymbol{n}=\mathbf{3 2}$ & $\boldsymbol{n = 6 4}$ \\
\hline 0.2 & $1.21 \mathrm{e}-3$ & $3.02 \mathrm{e}-4$ & $7.53 \mathrm{e}-5$ & $1.88 \mathrm{e}-5$ & $4.70 \mathrm{e}-6$ \\
0.4 & $2.43 \mathrm{e}-3$ & $6.03 \mathrm{e}-4$ & $1.51 \mathrm{e}-4$ & $3.76 \mathrm{e}-5$ & $9.41 \mathrm{e}-6$ \\
0.6 & $3.64 \mathrm{e}-3$ & $9.05 \mathrm{e}-4$ & $2.26 \mathrm{e}-4$ & $5.65 \mathrm{e}-5$ & $1.41 \mathrm{e}-5$ \\
0.8 & $4.86 \mathrm{e}-3$ & $1.21 \mathrm{e}-3$ & $3.01 \mathrm{e}-4$ & $7.52 \mathrm{e}-5$ & $1.88 \mathrm{e}-5$ \\
\hline
\end{tabular}


Table 4 Absolute errors for Example 3

\begin{tabular}{|c|c|c|c|c|}
\hline$x$ & $n=8, \sigma_{i}=0$ & $n=8, \sigma_{i}=1 / 2$ & $n=8, \sigma_{i}=1$ & $n=8, \sigma_{i}=r_{i}$ \\
\hline 0.2 & $1.14 \mathrm{e}-2$ & $1.12 \mathrm{e}-3$ & $1.02 \mathrm{e}-2$ & $5.88 e-4$ \\
\hline 0.4 & $2.27 e-2$ & $2.24 \mathrm{e}-3$ & $2.04 e-2$ & $1.18 \mathrm{e}-3$ \\
\hline 0.6 & $3.41 e-2$ & $3.36 e-3$ & $3.06 e-2$ & $1.76 e-3$ \\
\hline 0.8 & $4.54 \mathrm{e}-2$ & $4.48 e-3$ & $4.07 e-2$ & $2.35 e-3$ \\
\hline
\end{tabular}

\section{Conclusions}

Based on the idea of the integral mean value theorem and Newton iteration, a novel algorithm is constructed to solve the nonlinear Fredholm integral equations of the second kind. The convergence and the error of numerical results have been analyzed. By the obtained numerical results, we know the algorithm is feasible and valuable.

Authors' contributions

$\mathrm{HL}$ and $\mathrm{JH}$ were involved in the study design and manuscript preparation. Both authors read and approved the final manuscript.

\section{Acknowledgements}

The authors are grateful to the National Natural Science Foundation of China for financial funding under Grant number (11371079).

\section{Competing interests}

The authors declare that they have no competing interests.

Received: 7 December 2015 Accepted: 12 February 2016

Published online: 24 February 2016

\section{References}

Atkinson KE, Flores J (1993) Discrete collocation methods for nonlinear integral equations. IMA J Numer Anal 13:195-213 Atkinson KE, Potra F (1988) Discrete Galerkin methods for nonlinear integral equations. J Integral Eq. 13:17-54

Aziz I, Islam SU (2013) New algorithms for the numerical solution of nonlinear Fredholm and Volterra integral equations using Haar wavelets. J Comput Appl Math 239:333-345

Babolian E, Shahsavaran A (2009) Numerical solution of nonlinear Fredholm integral equations of the second kind using Haar wavelets. J Comput Appl Math 225:89-95

Lepik Ü, Tamme E (2007) Solution of nonlinear Fredholm integral equation via the Haar wavelet method. Proc Est Acad Sci Phys Math 56:17-27

Maleknejad K, Nedaiasl K (2011) Application of Sinc-collocation method for solving a class of nonlinear Fredholm integral equations. J Comput Math Appl 62:3292-3303

Ortege J, Kheinboldt w (1970) Iterative solution of nonlinear equations in several variables. Academic Press, New York Saberi-Nadjafi J, Heidari M (2010) Solving nonlinear integral equations in the Urysohn form by Newton-Kantorovichquadrature method. J Comput Math Appl 60:2058-2065

Zhong X-C (2013) A new Nyström-type method for Fredholm integral equations of the second kind. Appl Math Comput 219:8842-8847 